\title{
Estudiantes y medios tecnológicos: uso y actitud para la formación del lexicón
}

\author{
Klinge Villalba-Condori( ${ }^{(1) \star}$, Raidell Avello-Martínez ${ }^{(2)}$, Mario Berrios-Espezua ${ }^{(3)}$ y Sonia Castro-Cuba-Sayco(4) \\ (1) Universidad Continental, Arequipa, Perú, Avda. Los Incas s/n, José Luis Bustamante y Rivero, Arequipa, Perú. \\ (correo-e: kvillalba@continental.edu.pe) \\ (2) Universidad de Cienfuegos, Carretera a Rodas Km 4, Cienfuegos, Cuba. (correo-e: ravello@ucf.edu.cu) \\ (3) Universidad Católica de Santa María, Urb. San José s/n Umacollo, Arequipa, Perú. \\ (correo-e: mberrios@ucsm.edu.pe) \\ (4) Universidad Nacional de San Agustín de Arequipa, Perú, Santa Catalina 117, Arequipa, Perú. \\ (correo-e: sokli2512@gmail.com)
}

* Autor a quien debe ser dirigida la correspondencia.

Recibido Ago. 6, 2019; Aceptado Oct. 1, 2019; Versión final Ene. 2, 2020, Publicado Abr. 2020

\section{Resumen}

Este estudio tiene como objetivo demostrar la importancia del uso de los medios tecnológicos en relación con la calidad de lexicón de los estudiantes de la escuela secundaria. La investigación rompe con perfiles de investigación anteriores y se le consigna al lexicón un significado donde confluyen morfología, fonología y semántica. Se caracterizó primero la situación de los estudiantes en cuanto su lexicón, uso y actitud hacia la tecnología, encontrando que ciertamente los estudiantes que utilizan la tecnología y tienen una alta actitud hacia la tecnología logran desarrollar mejor su calidad de lexicón. Se encuentra alta correlación entre ellas usando la prueba Rho de Spearman. La investigación proporciona evidencias de cómo la formación de un lexicón de calidad está influenciada por la utilización de medios tecnológicos.

Palabras clave: lexicón; vocabulario; tecnología; aprendizaje; segunda lengua

\section{Students and technological media: use and attitude for lexicon formation}

\begin{abstract}
This study aims to demonstrate the importance of the use of technological media in relation to the lexicon quality of secondary school students. The research breaks with previous research profiles and lexicon is given a meaning where morphology, phonology and semantics converge. The study first characterized the situation of the students regarding their lexicon, use and attitude towards technology, and the calculation of their correlation, where it was discovered that certainly students who use technology and have a high attitude towards technology manage to develop better its lexicon quality. High correlation is found between them using the Rho-test of Spearman. The research provides evidence on how the formation of a quality lexicon was determined by the use of technological media.
\end{abstract}

Keywords: lexicon; vocabulary; technology; learning; second language 


\section{INTRODUCCIÓN}

Esta investigación busca determinar la dependencia que existen entre el uso de medios tecnológicos y la actitud hacia la tecnología con la calidad del lexicón de los estudiantes de segundo de secundaria. Por otro lado, cabe recalcar que no se está utilizando el concepto de vocabulario, muy común de la lingüística estructural, sino el de lexicón, propio de Chomsky (1979) desde la Teoría Estándar Extendida. En tal sentido, Chomsky (1979) propone este término como transversal a la estructura del lenguaje, pues tiene representación gráfica, como fonológica y semántica; además, es piedra angular para la construcción de sentencias que dan ser a la lengua. Según la RAE el Lexicón es el conocimiento léxico que un hablante posee sobre una lengua, es decir el repertorio o diccionario o palabras que se hacen común en su uso diario.

Teniendo en cuenta los rasgos cuasi computacionales del lexicón, pues, la estructura de este en nuestro cerebro es parecida a una base de datos que se alimenta día a día y rápidamente con las palabras que escuchamos cotidianamente para luego ser usadas en la formación de estructuras (sentencias) que darán como resultado al texto (Birchenall y Müller, 2014). Los medios tecnológicos entran como parte importante del aprendizaje de la lengua en la cotidianeidad del estudiante, por ello en los siguientes párrafos se revisarán investigaciones en relación al uso o actitudes hacia la tecnología en el proceso de aprendizaje, e inclusive en la obtención de un buen lexicón al que llaman vocabulario comúnmente.

Es vital reconocer la gran utilidad de los medios tecnológicos en el desarrollo del lexicón, pero, es mayor el estudio que se le hace en la adquisición de una L2. Por ello, es necesario que se amplíen los estudios del uso de las tecnologías para el desarrollo de la L1, pues, el conocimiento de la perspectiva semántica y fonológica del lenguaje proporciona las estructuras del significado y sobre todo permite que el estudiante pueda elevar su expresión y poder dar un fondo pragmático a su conversación; es decir, que pueda lograr comunicar la intención comunicativa de los textos orales o escritos que pueda producir. Además, estudiar el lexicón permite engrosar las líneas de la didáctica de la lengua, herramienta vital para los procesos de enseñanza y aprendizaje a dominar por los educadores.

Por un lado, la investigación pretende dar solución a la interrogante de cómo la formación de un lexicón de está influenciada por la utilización de medios tecnológicos, se complementa este análisis con la actitud hacia el uso de tecnología, es decir que los estudiantes sujetos de investigación son aquellos cuya actitud es constante para el uso de tecnologías, con las características del lexicón, como cúmulo de fonemas, lexemas y semas almacenados en el cerebro de los estudiantes, la investigación cobra relevancia al proponer un estudio en relación con la profundización de la competencia comunicativa a través del lexicón por medio de la tecnología, yendo, de cierta forma, en contra de la corriente de investigaciones que se ocupan mayormente del vocabulario, esta competencia es parte del diseño curricular nacional que utiliza la Educación Básica Regular para el Área Curricular de Comunicación.

El estudio de Leiva (2014) tuvo como fin conocer las actitudes de los docentes hacia el uso de las Tecnologías de la Información y de la Comunicación (TIC) en relación con las características laborales y de capacitación de los mismos. Para la investigación tomaron como muestra a 139 docentes de instituciones públicas del distrito de la Merced de la provincia de Chanchamayo, departamento de Junín (Perú) a los cuales se les aplicó un cuestionario con 30 ítems. La investigación determinó que los docentes muestran una mala actitud ante el uso de tecnologías en su trabajo administrativo; pero, si estas se utilizan para el aprendizaje de los estudiantes, se encuentra una mejor actitud en cuanto su uso.

La investigación de Lloclla y Rojas (2015) tuvo como objetivo encontrar las relaciones entre el uso de las TIC por los estudiantes y el proceso de enseñanza aprendizaje; se tomó como muestra a 73 estudiantes matriculados en el Centro de Educación Técnico Productiva "Pedro Paulet" del departamento de Huancavelica (Perú) y se les aplicó un cuestionario de 45 preguntas; en consecuencia, la pesquisa mostró que los estudiantes que utilizan las TIC logran un mejor desarrollo en el proceso de enseñanza y aprendizaje. EI estudio de Alzahrani (2015) propone dilucidar la importancia que tienen los dispositivos móviles en el momento de adquirir un buen vocabulario en el aprendizaje de una lengua extranjera; en este sentido, se realizó una revisión documental; en consecuencia, se determinó que la mayoría de los estudios sobre usos que se le da a la tecnología está en relación a la adquisición de vocabulario.

Otros resultados interesantes se encuentran en la pesquisa de Janjic et al. (2016) que tuvo el fin de conocer la actitud que tienen los estudiantes croatas ante el uso de tecnología en el aprendizaje de idiomas asiáticos en cuanto el vocabulario y gramática; en este sentido, se tomó una muestra de 203 estudiantes pertenecientes a los programas de idiomas asiáticos (hindi, japonés, coreano y sánscrito) en las ciudades Zagrep, Rijeka, Varazdin y Split (Croacia), aplicándoles un cuestionario; en consecuencia, se identificó que los estudiantes presentan una buena actitud hacia el uso de tecnologías en el aprendizaje, pues, algunos utilizan estos medios para aprender. 


\section{OTROS ANTECEDENTES}

Por una parte, Gürocak (2016), en su investigación propuso conocer si la utilización de los mensajes de texto son en verdad útiles para optimizar el aprendizaje del vocabulario en inglés; para este se seleccionaron 56 estudiantes del programa de inglés de pregrado de una universidad estatal de Anatolia (Turquía), para aplicarles diversos instrumentos como la Vocabulary Knowledge Scale, un cuestionario actitudinal y un conjunto de preguntas de entrada y salida sobre el uso de teléfonos móviles en el aprendizaje de vocabulario en inglés; el estudio mostró que los estudiantes utilizan sus teléfonos móviles como instrumentos eficaces para el aprendizaje del vocabulario en inglés; además, se logró demostrar que mientras los estudiantes tengan una actitud positiva hacia el uso de los móviles para el aprendizaje se obtendrá un mejor vocabulario.

Cojocnean (2016) tuvo como objetivo determinar la relación entre las actitudes y uso de medios tecnológicos en el aprendizaje del vocabulario de una segunda lengua; por ello, acudiendo a métodos mixtos, cuantitativo y cualitativo, seleccionó una muestra de 1239 y otra no probabilística de 43 estudiantes pertenecientes a 9 instituciones secundarias de Rumania, aplicándoles un cuestionario de 14 preguntas y focus group; de esta manera, se mostró que, pese a que todos los estudiantes cuentan con un dispositivo móvil, existe una actitud neutra en el uso de dispositivos móviles para el aprendizaje de vocabulario producto de la falta motivación e inserción de la tecnología en las escuelas, pues estos dispositivos están prohibidos. De Van Hove et al. (2017) propuso comprobar la relación que existe entre la utilización de un medio tecnológico como es la tablet para el aprendizaje de vocabulario de una segunda lengua; en este sentido, utilizando el método cuantitativo, se escogió una muestra de 282 estudiantes de tres colegios de Flandes (Bélgica) y se les aplicó tres pruebas sobre vocabulario compuesta por 36 palabras con un ascendente nivel de complejidad; de esta manera, se determinó la influencia del uso de medios tecnológicos, pues luego de un mínimo de 10 días utilizando las tablet, los estudiantes adquirieron vocabulario en una segunda lengua, para este caso el francés.

Hernández y Tayo (2017) en su investigación tiene como objetivo determinar las relaciones que existen entre los medios tecnológicos y el aprendizaje de una segunda lengua (inglés) de forma semipresencial y la satisfacción docente en el uso de la tecnología; por ello, se tomó una muestra de 399 estudiantes y 14 docentes pertenecientes al Centro de Idiomas de la Universidad Técnica de Ambato (Ecuador), a los cuales se les aplico un cuestionario de encuesta de 37 ítems para docentes y 33 ítems para los estudiantes; de esta forma, se determinó que el uso de medios tecnológicos virtuales como las plataformas ayudan grandemente a la obtención de competencias comunicativas, por ejemplo un buen vocabulario, en una segunda lengua además de encontrar una buena actitud por parte de los docentes como estudiantes hacia estas tecnologías.

De igual forma, el trabajo de Stošić y Fadiya (2017) propone comprobar la relación entre las actitudes de los estudiantes y el uso de TIC en su proceso de formación educativa; por ende, utilizando el método cuantitativo, se tomó una muestra de 285 estudiantes de primero a cuarto año de la Universidad Americana de Girne (Turquía) y se les aplicó un cuestionario de encuesta de 45 ítem; en consecuencia, se identificó que los estudiantes utilizan TIC pero no piensan que el uso de estos medios electrónicos sean de vital importancia, más bien, existe un equilibrio entre uso de TIC y sus clases presenciales y, aunque les es importante tener presencialmente al docente, son conscientes de la ayuda de los medios tecnológicos en su aprendizaje. Jiménez et al. (2017) buscó encontrar la influencia de los medios tecnológicos, en este caso la tablet y aplicaciones IOS, Android, en la adquisición del lenguaje desarrollo de la comunicación en un niño con trastorno del espectro autista (TEA); es así, utilizando el método cualitativo, se escogió a un niño con TEA de cuatro años del CEIP San José de Calasanz de Yecla en Murcia (España), de quien se hizo el estudio utilizando un cuestionario de 42 ítem; en este sentido, se determinó que producto de la utilización de aplicaciones de la tablet se logró motivación y captura de la atención del niño logrando mejorar ampliamente sus capacidades comunicativas.

Yang y Yin (2018) propuso demostrar la eficacia de uso de tecnología móvil en el aprendizaje de coloquialismos en estudiantes de chino como segunda lengua; se tomó una muestra de 46 estudiantes de chino hablantes de inglés como lengua materna pertenecientes a dos universidades del medio oeste de Estados Unidos y se les aplicó una Escala de Conocimiento de Vocabulario y un cuestionario sobre WeChat de 11 ítem, la investigación mostró que la cotidianeidad del uso de medios móviles como el teléfono móvil por parte de los estudiantes logra desarrollar el vocabulario de una segunda legua en un tiempo corto además de ser una herramienta útil en todos los niveles del aprendizaje de una L2. De igual manera, la investigación de Che et al. (2018) tuvo como objetivo desarrollar un sistema de Realidad Aumentada (AR) para mejorar el vocabulario integrando a este la ortografía y la fonología utilizando un sistema de reconocimiento del habla; estos autores tomaron una muestra de 30 estudiantes de distintas escuelas primarias de Malasia y se les aplicó un cuestionario llamado System Usability Scale (SUS) de cara a la satisfacción con la AR; en consecuencia, se identificó una calificación SUS alta; es decir, los estudiantes estaban muy satisfechos con la aplicación, y los docentes, a los cuales se entrevistó, muestran la misma actitud, además, de mostrar que el reconocimiento ayudó en el vocabulario y en la correcta pronunciación. En otro contexto, Zhou y Wei (2018) se propusieron una revisión de la literatura sobre la adquisición de segundas lenguas a través de medios 
tecnológicos; entonces, utilizando el método cualitativo, revisaron investigaciones desde 1998 hasta el 2017; llegando a la conclusión de que desde 1980 la tecnología ha ido cambiando la forma de enseñar una segunda lengua, pues, las tecnologías 3D, realidad aumentada y reconocimiento de voz han logrado mejorar los resultados en los ámbitos de vocabulario, escritura, lectura.

Xinyuan et al. (2018) en su estudio se pretende explicar el proceso de inserción de la tecnología en la enseñanza de la lectura y los cambios teóricos sobre la lectura en el tránsito de la escuela tradicional a los entornos digitales; en este sentido, se optó por utilizar la revisión documental, y se analizaron 70 artículos sobre enseñanza de la lectura publicados en Florida (Estados Unidos) durante los años 2004 y 2015; la pesquisa identificó que existe poca conexión entre la teoría sobre la lectura y la práctica desde las tecnologías, aunque la tecnología sea eficaz para la enseñanza de la lectura, existen pocas investigaciones que relacionen estas tecnologías con la teoría de la lectura. La investigación de Çetinkaya y Soner (2018) tuvo como objetivo demostrar la influencia de las redes sociales Facebook y WhatsApp en la enseñanza del vocabulario de la lengua inglesa; por ello, empleando métodos mixtos, se realizó un muestro por criterio, eligiendo una escuela secundaria estatal con un grupo de 93 estudiantes que cumplían todos los criterios para la aplicación de los test (prueba de 70 ítem) y de 62 para el grupo experimental, que luego de las pruebas, se les pidió su opinión para el análisis cualitativo; en consecuencia, se identificó la influencia de estas redes sociales en el aprendizaje del vocabulario, pues, los estudiantes que usaron WhatsApp en su aprendizaje lograron cambios sustanciales sus calificaciones.

\section{METODOLOGÍA}

El método utilizado en la investigación es de corte cuantitativo, con un diseño de investigación relacional, pues se busca explicar la relación entre las variables independientes Uso de Medios Tecnológicos y Actitud a los Medios Tecnológicos y la variable dependiente Calidad de Lexicón.

\section{Participantes}

Los participantes en esta investigación fueron 68 estudiantes elegidos aleatoriamente dentro de los cuales se encuentran 38 hombres $(55,8 \%)$ y 30 mujeres $(44,2 \%)$, como se muestra en la Tabla 1 . La cantidad de la muestra es resultante del cálculo de una muestra probabilística a una población de 164 estudiantes pertenecientes a las secciones A, B, C, D, E y F del $2^{\circ}$ año de educación secundaria de la Institución Educativa del nivel secundario de la Educación Básica Regular de Perú. El cálculo de la muestra fue al $95 \%$ del nivel de confianza y 5\% como margen de error, encontrando 116 estudiantes. Posteriormente se realizó el ajuste de la muestra, encontrando la muestra ajustada de 68 estudiantes.

Tabla 1: Distribución de los participantes

\begin{tabular}{|c|c|}
\hline Sexo & Participantes \\
\hline Hombres & 38 \\
\hline Mujeres & 30 \\
\hline Total & 68 \\
\hline
\end{tabular}

\section{Instrumentos}

Los instrumentos utilizados en la investigación están en relación con cada una de las variables a analizar; en este sentido, el instrumento para medir la calidad de lexicón se adaptó del estudio de Quesada, Escurra y Delgado (2001), quienes validaron el mismo. Este instrumento es una Prueba de Riqueza Léxica; es decir, mide el nivel de lexicón de los estudiantes a partir de 26 ítems que deben ser contestados en 5 minutos; cada ítem contiene una palabra o frase generadora; como, por ejemplo, si se propone la palabra árboles la respuesta está llevada a dos especies de la misma: álamo, sauce; o artes: música, pintura. Esta prueba tiene una puntuación de 0 a 52 puntos y cada pregunta está codificada de manera que si el estudiante no responde la pregunta no valdrá nada (Sin respuesta $=0$ ), si responde una palabra o palabras sinónimas de la palabra generadora o entre sí se valorará con un punto (1 palabra o sinónimas=1), pero, la respuesta son dos palabras especie de la generadora se valorará con dos puntos (2 palabras=2). Además, esta prueba consta con una escala de tres niveles; el nivel bajo consta de puntajes partiendo de 0 a 27, el nivel medio de 28 a 35 , y el nivel alto de 36 a 52. Por otra parte, el resultado del alfa de Cronbach del instrumento fue de 0.877 , lo cual demuestra tener una buena confiabilidad, según parámetros de esta área de la ciencia (Creswell, 2012).

Para medir el uso de medios tecnológicos y actitud hacia la tecnología se usaron otros instrumentos; los cuales, fueron adaptados de los cuestionarios de la investigación de Conde Antuca (2017). Estos cuestionarios son traducidos en dos test, el primero mide la variable uso de medios tecnológicos, pues, las preguntas están divididas en dos grupos, el primero constituido por 18 ítems (del 1 al 18) referidos a uso de medios físicos como celulares digitales y laptops, y su uso; el otro, por 22 Ítems (del 19 al 40) sobre el uso de 
software como Word, Excel, email, Facebook, WhatsApp, y el interés por aprender el uso de los mismos. Este test tiene una escala, de 40 a 200 puntos, tipo Likert con cuatro respuestas ordenadas por: nunca $=1$, raramente $=2$, ocasionalmente $=3$, frecuentemente $=4$, y muy frecuentemente $=5$. Además, el baremo de este instrumento está estructurado por niveles: bajo de 40 a 93, medio de 94 a 147, y alto de 148 a 200. Así mismo, el resultado del alfa de Cronbach del instrumento fue de 0.928 demostrando tener una alta confiabilidad (Creswell, 2012).

El test de actitud hacia la tecnología está dividido en tres grupos de preguntas; primero, está el afectivo con preguntas de satisfacción o si se sienten bien usando medios tecnológicos físicos como virtuales, los ítems son 10 (del 1 al 10); segundo, preguntas cognitivas, relacionadas al conocimiento de medios físicos como virtuales, con 10 Ítems (del 11 al 20); tercero, preguntas conductuales, relacionadas al buen uso de medios físicos o virtuales, con 8 ítems (del 21 al 28). Este test también tiene una escala, de 28 a 140 puntos, tipo Likert con cuatro respuestas ordenadas por: nunca $=1$, raramente $=2$, ocasionalmente $=3$, frecuentemente $=4$, y muy frecuentemente $=5$. Además, el baremo de este instrumento está estructurado por niveles: bajo de 28 a 70 , medio de 71 a 110, y alto de 111 a 140 . Así mismo, el resultado del alfa de Cronbach del instrumento fue de 0.924 demostrando tener una alta confiabilidad (Creswell, 2012).

\section{Procedimiento}

La aplicación de la prueba como de los test se realizó en un solo día, en el cual los estudiantes recibieron refuerzo educativo; por ende, se combinaron todas las secciones y se procedió a entrar a tres secciones aleatoriamente para aplicar los tres instrumentos por estudiante, la prueba de lexicón duró 5 minutos, los otros dos test 20 minutos cada uno. Es decir, se aplicó los instrumentos al comenzar la jornada educativa con una duración total de 45 minutos; pues, en esos momentos los estudiantes se encuentran en solaz y más solícitos para llenar los instrumentos sin premuras que se traduzcan en grandes sesgos de información. Luego de aplicado el instrumento se procedió a recoger los instrumentos, en el caso de la prueba de lexicón, para su posterior calificación y codificación. Luego de tal calificación y codificación de la prueba léxica, esta, junto con los demás instrumentos, fueron procesados en el software SPSS (Ver. 20).

\section{RESULTADOS Y DISCUSIÓN}

Se comenzará por denotar las características generales por cada una de las variables, para luego pasar al análisis de las relaciones entre las mismas. La primera variable a resaltar es la de calidad de lexicón en los estudiantes de la IE San Martín de Socabaya, de estos el $27.9 \%$, tienen un nivel bajo; el nivel medio detenta $23.5 \%$, en el nivel alto encontramos un $48.5 \%$; es decir, que el porcentaje acumulado más importante (76.4\%) pertenece a los estudiantes de nivel medio y alto. En cuanto al uso de medios tecnológicos, encontramos un $8.8 \%$ de estudiantes en el nivel bajo, el $72.1 \%$ en el nivel medio, y el $19.1 \%$ en el nivel alto, teniendo un acumulado de estudiantes con un nivel medio a alto de $91.2 \%$. En los niveles de actitud hacia la tecnología encontramos el $7.4 \%$ de estudiantes en el nivel bajo; el $69.1 \%$, en el nivel medio; y el $23.5 \%$ en el nivel alto; teniendo un aculado de $91.6 \%$ en los niveles medio y alto. También se debe tener en cuenta que el nivel que más prevalece en los test es el medio, mientras que en la prueba es el nivel alto el que tiene un porcentaje mayor.

Tabla 2: Uso de medios tecnológicos por calidad de lexicón.

\begin{tabular}{|l|l|l|l|l|}
\hline & \multicolumn{4}{|c|}{ Nivel de calidad del lexicón } \\
\hline \multirow{2}{*}{$\begin{array}{l}\text { Nivel de uso } \\
\text { de medios } \\
\text { tecnológicos }\end{array}$} & & Bajo & Medio & Alto \\
\cline { 2 - 5 } & Bajo & $21.1 \%$ & $6.3 \%$ & $3.0 \%$ \\
\cline { 2 - 5 } & Medio & $68.4 \%$ & $87.5 \%$ & $66.7 \%$ \\
\cline { 2 - 5 } & Alto & $10.5 \%$ & $6.3 \%$ & $30.3 \%$ \\
\hline
\end{tabular}

Luego de haber descrito las características generales de cada variable pasaremos a conocer las asociaciones entre ellas; por ello usando la prueba de Chi cuadrado de Pearson para las variables uso de medios tecnológicos y calidad de lexicón se encontró $(X 2=9, g l=4, p=0.048)$ que existe asociación entre estas; además que de acuerdo a sus porcentajes (tabla 2) evidencian los porcentajes más altos en el cruce de los niveles medios de ambas variables, además se encontró que al acumular los porcentajes entre variables estas van en ascenso; en este sentido, los estudiantes que tienen un nivel bajo de calidad de lexicón, el $89.5 \%$ tienen un nivel de bajo a medio de uso de medios tecnológicos; los que tienen un nivel medio de lexicón, el $87.5 \%$ tienen también un nivel medio de uso de medios tecnológicos; así mismo, como se puede apreciar, quienes tienen un nivel alto de lexicón, el 96.9\% tienen un nivel medio alto de uso de medios tecnológicos. 
Tabla 3: Actitud hacia la tecnología por calidad de lexicón.

\begin{tabular}{|l|l|l|l|l|}
\hline \multirow{2}{*}{$\begin{array}{l}\text { Nivel de } \\
\text { actitud hacia } \\
\text { la tecnología }\end{array}$} & \multicolumn{4}{|c|}{ Nivel de calidad del lexicón } \\
\cline { 2 - 5 } & Bajo & Bajo & Medio & Alto \\
\cline { 2 - 5 } & Medio & $68.4 \%$ & $6.3 \%$ & $0.0 \%$ \\
\cline { 2 - 5 } & Alto & $10.5 \%$ & $12.5 \%$ & $36.4 \%$ \\
\hline
\end{tabular}

Para las variables actitud hacia la tecnología y calidad de lexicón también se aplicó la prueba de Chi cuadrado de Pearson, cuyo resultado $(X 2=12.291, g l=4, p=0.015)$ demuestra que existe asociación entre las variables. Por ello, teniendo en cuenta la tabla 3 se determina que los porcentajes relacionados van ascendiendo, por ejemplo, los estudiantes que tienen un nivel bajo de calidad de lexicón, el $85.5 \%$ poseen niveles de bajo a medio de actitud hacia la tecnología; para el nivel medio de lexicón, el $81.3 \%$ posee también un nivel medio de actitud hacia la tecnología; y para el nivel alto de lexicón, el 100.0\% tiene un nivel de medio a alto de actitud hacia la tecnología.

En la exploración de las variables se encontró que la variable calidad de lexicón se comporta de forma no paramétrica (Kolmogorov-Smirnov $=0.155, \mathrm{gl}=68, \mathrm{p}<0.000$ ) al igual que la variable uso de medios tecnológicos (Kolmogorov-Smirnov=0.108, $g=68, p=0.049$ ); sin embargo, la variable actitud hacia la tecnología tiene un comportamiento paramétrico (Kolmogorov-Smirnov=0.083, $\mathrm{gl}=68, \mathrm{p}=0.200$ ). Por ello, que luego de aplicar la prueba U de Mann Whitney, las variables de lexicón $(p=0.376)$ y uso de la tecnología $(p=0.966)$ en relación con el sexo no se hallaron diferencias significativas; así mismo, en la variable actitud hacia la tecnología (t de student=-0.106, $g \mathrm{l}=66, \mathrm{p}=0.916$ ), como se puede apreciar, tampoco existe diferencia entre los sexos. Este resultado revela la no importancia respecto al sexo.

Para el análisis de la correlación entre las variables, se tiene en cuenta que la mayoría de las variables son no paramétricas, incluyendo la variable dependiente calidad de lexicón, por ello, se utilizó pruebas no paramétricas para la correlación. En este sentido, para las variables uso de medios tecnológicos y calidad de lexicón se tiene como resultado de la prueba Rho de Spearman un coeficiente $=0.384$ y $p=0.001$, este demuestra que existe una fuerte correlación entre estas variables; las variables actitud hacia la tecnología y calidad de lexicón pasaron por la misma prueba arrojando un coeficiente $=0.278$ y $p=0.022$, demostrando también, la existencia de correlación entre ellas, confirmando así, que el uso de medios tecnológicos y la actitud hacia la tecnología determinan el desarrollo de un mejor vocabulario en los estudiantes del segundo año de secundaria, sin importar el sexo.

La investigación busca en un primer momento revisar las investigaciones sobre el uso de las TIC en el aprendizaje del vocabulario y encontrar similitudes con nuestros resultados. La primera está relacionada al uso de medios como demás tecnologías en pro del aprendizaje en sí mismo como del vocabulario de una segunda lengua. En tal sentido, es preciso destacar a Hernández y Tayo (2017) quienes, en correspondencia con nuestra investigación, estudiaron las relaciones que existen entre los medios tecnológicos y el aprendizaje de una segunda lengua (inglés) y encontraron que el uso de medios tecnológicos virtuales como las plataformas ayudan grandemente a la obtención de competencias comunicativas, por ejemplo, un buen vocabulario, en una segunda lengua además de encontrar una buena actitud por parte de los docentes, así como estudiantes hacia estas tecnologías. Ciertamente la tendencia en el mundo se dirige al uso de tecnologías para las segundas lenguas, pero, no existen muchas investigaciones de cara al aprendizaje del vocabulario de la lengua vernácula.

Posiblemente se dé este problema por las definiciones de vocabulario que se utilice, pues, existen distintas posiciones teóricas, basadas en investigación científica, desde la lingüística, para este tópico y por tal motivo puede introducir confusión al respecto. La perspectiva de la investigación cambia el continuum del uso de las tecnologías de la información y las comunicaciones, pues, esto que se conoce como vocabulario para la investigación se llama Lexicón, término tomado de la lingüística generativa y que en realidad encierra todo el significado de lo conocido como vocabulario. En este sentido, Chomsky (1979) al decir lexicón propone una unidad básica dotada de características morfológicas, semánticas y fonológicas que la convierten en piedra angular para la formación de la competencia gramatical y así crear las estructuras sintácticas que construirán cualquier lengua. En este sentido, se divisa la necesidad de adquirir un buen lexicón no sólo para otras lenguas, sino, para todas las que se posea, especialmente la vernácula, presente siempre en las instituciones educativas. En concordancia con lo anterior, el estudio ha demostrado que la tecnología ayuda a la mejora de la calidad de lexicón. 
Teniendo en cuenta que la relación entre el uso de medios tecnológicos y lexicón es más fuerte que la actitud que se tiene hacia ellos, es provocada porque los estudiantes dedican una gran parte del tiempo a utilizar estos medios como instrumentos distractores, es más, ya usan estos instrumentos; por ende, su uso se convierte en cotidiano modificando la actitud hacia ellos. Sin embargo, como también plantea Cojocnean (2016), estos medios al sirven de comunicación, pero transforman el lexicón, pues, existe libertad en su uso, modificación que no siempre es beneficiosa para construir sentencias bien estructuradas. Esto lograría en el estudiante el conocimiento de neologismos propios de la comunicación por web que en cierta forma afecta el buen desarrollo del lexicón, teniendo como consecuencia que, pese a que se demuestra la utilidad de tecnología para el desarrollo del lexicón, la mayoría de estudiantes detentan un nivel medio del mismo.

Otra situación importante de reconocer es que los estudiantes tienen un buen nivel de lexicón, lo que nos hace ver la necesidad de conocer más palabras para utilizar en la comunicación, pues este es el fin de los medios tecnológicos, servir de medios de comunicación entre personas, profesores estudiantes y hasta niveles como lograr comunicar el conocimiento del docente al estudiante, para hacer posible la socialización de tal conocimiento. La investigación demostró que los estudiantes tienen un buen nivel de calidad del lexicón, pero es necesario contrastar este nivel con las demás variables. Es decir, que la asociación entre niveles revela que ciertamente la tecnología es un gran potenciador, como también se constató en Lloclla y Rojas (2015), Gürocak (2016), Van Hove et al. (2017) y Yang y Yin (2018), pues, mientras más crecía la presencia de estudiantes con buen vocabulario, estos mismos estudiantes aparecían con mejores resultados en los otros test aplicados en el estudio, es decir, usa más frecuentemente medios tecnológicos y, como se esperaba, tuvieron una buena actitud hacia ellos.

\section{DISCUSIÓN FINAL}

La investigación sugiere la veracidad del núcleo de su ser. Es decir, muestra que los estudiantes de segundo año, que tienen uso de medios tecnológicos y una buena actitud hacia la tecnología logran alcanza niveles más altos de calidad de lexicón. En otras palabras, usar la tecnología con buena actitud logra aumentar el caudal de voces que constituye el lexicón de cada estudiante, resultados similares encontraron en Janjic et al. (2016). Además, demuestra que la tecnología es eficaz para la enseñanza de la lectura, sobre lo cual existen pocas investigaciones que relacionen estas tecnologías con la teoría de la lectura, como también fue comprobado por Van Hove et al. (2017), quienes encontraron relación entre la utilización de un medio tecnológico y el aprendizaje del vocabulario de una segunda lengua.

Por tales razones, es de gran importancia que se utilicen medios tecnológicos en las aulas, sin restricciones, pero a la vez se encamine hacia el aprendizaje y buen uso de la lengua vernácula y no solo pensar en la adquisición de voces extranjeras, sino también, mejorar la articulación de la lengua vernácula. En este caso podríamos decir que los profesores, así como las autoridades políticas, están llamados permitir que estos estudiantes que han demostrado habilidades en el uso de la tecnología sean capaces de usarla en la construcción de una buena competencia comunicativa.

A partir de los resultados obtenidos, la investigación proporciona evidencias de cómo la formación de un lexicón de calidad estuvo determinada por la utilización de medios tecnológicos, y su relación con la actitud de los estudiantes hacia la tecnología; en correspondencia con las características del lexicón, como cúmulo de fonemas, lexemas y semas almacenados en el cerebro de los estudiantes. Por otro lado, la investigación aporta al campo de estudio, al proponer un estudio en relación con la profundización de la competencia comunicativa a través del lexicón por medio de la tecnología.

\section{CONCLUSIONES}

De acuerdo al trabajo presentado y a los resultados obtenidos, se pueden plantear las siguientes conclusiones principales:

1.- El Lexicon está influenciado necesariamente por la actitud hacia la tecnología y por el uso mismo de las tecnologías, se encontró una correlación fuerte para ambos casos.

2.- Los sujetos de estudio tienen una inclinación recurrente y constante respecto al uso de tecnologías, se complementó este análisis con el factor sexo, encontrándose semejanzas respecto al uso de Lexicon, es decir no existiendo diferencias, aquí se evidencia la no relevancia de este factor.

3.- La construcción del Lexicon se ve influenciada por el contexto en el cual se encuentra inmerso, la investigación reafirma lo encontrado anteriormente sobre la construcción del Lexicon a partir de la interacción en este caso con medios y sobre todo con quien se comunica en este caso los estudiantes de la Educación Básica Regular. 


\section{AGRADECIMIENTOS}

Este artículo ha sido elaborado en el marco del proyecto de mejora de Habilidades Comunicativas en Arequipa conjuntamente con la sociedad civil y la Municipalidad Provincial de Arequipa a quienes estamos profundamente agradecidos por su financiamiento.

\section{REFERENCIAS}

Acebedo-Afanador, M.J., Aznar-Díaz, I., y Hinojo-Lucena, F.J., Instrumentos para la Evaluación del Aprendizaje Basado en Competencias: Estudio de caso. Información tecnológica, 28(3), 107-118 (2017).

Alzahrani, H., Examining the Effectiveness of Utilizing Mobile Technology in Vocabulary Development for Language Learners. http://doi.org/10.2139/ssrn.2834731, Arab World English Journal, 6(3), 108-119 (2015).

Birchenall, L. B., y Müller, O., La Teoría Lingüística de Noam Chomsky: del Inicio a la Actualidad. Lenguaje, 42(2), 417442 (2014).

Çetinkaya, L., y Sütçü, S.S., The Effects of Facebook and WhatsApp on Success in English Vocabulary Instruction. http://doi.org/10.1111/jcal.12255, Journal of Computer Assisted Learning, 34, 504-514 (2018).

Che Hashim, N., Abd Majid, N., y Khalid Obeidy, W., User Satisfaction for an Augmented Reality Application to Support Productive Vocabulary Using Speech Recognition. http://doi.org/10.1155/2018/9753979, Advances in Multimedia (2018).

Chomsky, N., Introducción a la Teoría Estándar Extendida. In N. ChomsKy, J. Emonds, J., Faye, R., Jackendoff, J. Milner, C., Otero, F., Selkirk (Eds.), La Teoría Estándar Extendida (pp. 19-42). Madrid (1979).

Cojocnean, D., Factors Determining Students' Low Usage of Mobile Tools in their English Vocabulary Learning. Monográfico I, 31-43 (2016).

Conde Atunca, F., Actitud docente y Uso de la Tecnología de la Información y Comunicación en Instituciones Educativas Públicas de Comas, Universidad César Vallejo (2017). Recuperado de: http://repositorio.ucv.edu.pe/handle/UCV/8497

Creswell J., Educational research: Planning, conducting, and evaluating quantitative and qualitative research (4ta ed). Pearson Education, Boston (2012).

Eco, U., Apocalípticos e integrados. Palabra en el tiempo. Ensayo, 39. España: Editorial Lumen (1965).

Gürocak, F., Learning Vocabulary through Text Messages: Help or Distraction? http://doi.org/10.1016/j.sbspro.2016.10.086, Procedia - Social and Behavioral Sciences, 232, 623-630 (2016).

Hernández, E., y Tayo, E., La Satisfacción de los Docentes y Estudiantes en una Experiencia de Aprendizaje Apoyada en la Tecnología. Revista Publicando, 8, 188 (2017).

Janjic, M., Librenjak, S. y Kocijan, K., Croatian Students' Attitudes towards Technology Usage in Teaching Asian Languages - A field research. http://doi.org/10.1109/MIPRO.2016.7522276, MIPRO, 946-950 (2016).

Jiménez, M., Serrano, J., y Prendes, M., Estudio de Caso de la Influencia del Aprendizaje Electrónico Móvil en el Desarrollo de la Comunicación y el Lenguaje con un Niño con TEA. http://doi.org/10.5565/rev/educar.782, Educar, 532 (2017).

Leiva Huamanyalli, D. L., Actitudes hacia las TIC en Docentes de Educación Básica Regular de la Merced - Chanchamayo. Universidad Nacional del Centro del Perú (2014).

Lloclla, M., y Rojas, W., Las Tecnologías de la Información y Comunicación en el Proceso de Enseñanza-Aprendizaje de los Estudiantes del Centro de Educación Técnico Productiva "Pedro Paulet" de Huancavelica. Tesis de pregrado, Universidad Nacional de Huancavelica, Perú (2015).

Quesada, M. R., Escurra, M., y Delgado, M.A., Riqueza Léxica En Estudiantes De Educación Básica (Nivel Secundaria) De Lima Metropolitana. https://doi.org/10.15381/rinvp.v4i1.5013, Revista de Investigación en Psicología, 1, 109-119 (2001).

Stošić, L., y Fadiya, S., The Attitudes of Students towards the Use of ICT during their Studies, https://doi.org/10.21702/rpj.2017.1.9, Revista Psicológica Rusa, 14(1), 135-148 (2017).

Van Hove, S., Vanderhoven, E., y Cornillie, F., The tablet for Second Language Vocabulary Learning: Keyboard, Stylus or Multiple Choice, https://doi.org/10.3916/C50-2017-05, Comunicar, 25(50), 53-63 (2017).

Xinyuan, Y., Kuo, L.J., Ji, X., y McTigue, E.A., A Critical Examination of the Relationship Among Research, Theory, and Practice: Technology and Reading Instruction, https://doi.org/10.1016/j.compedu.2018.03.009, Computers and Education, 125(March), 62-73 (2018).

Yang, J., y Yin, C., Learning Chinese Colloquialisms through Mobile Technology. Journal of Technology and Chinese Language Teaching, 9, 239-256 (2018).

Zhou, Y., y Wei, M., Strategies in Technology-Enhanced Language Learning, https://doi.org/10.14746/ssllt.2018.8.2.13, Studies in Second Language Learning and Teaching, 8(2), 471-495 (2018). 